\title{
Epidemiological evaluation of cats associated with feline polycystic kidney disease caused by the feline PKD1 genetic mutation in Japan
}

\author{
Reeko SATO ${ }^{1) *}$, Naohiro UCHIDA ${ }^{1)}$, Yuka KAWANA ${ }^{1)}$, Minako TOZUKA ${ }^{1}$, \\ Saori KOBAYASHI ${ }^{1)}$, Nana HANYU1), Yoshinobu KONNO'1), Aiko IGUCHI ${ }^{1)}$, \\ Yayoi YAMASAKI ${ }^{1}$, Konomi KURAMOCHI ${ }^{1)}$ and Masahiro YAMASAKI ${ }^{1)}$ \\ ${ }^{1)}$ Department of Veterinary Internal Medicine, Iwate University, 3-18-8 Ueda, Morioka-shi, Iwate 020-8550, \\ Japan
}

J. Vet. Med. Sci.

81(7): 1006-1011, 2019

doi: 10.1292/jvms.18-0309

Received: 6 June 2018

Accepted: 8 May 2019

Advanced Epub:

3 June 2019

\begin{abstract}
Feline polycystic kidney disease (PKD), an inherited autosomal dominant disease, has been reported to occur mostly in Persian or Persian related cats, and to be associated with a mutation from C to A at position 10063 in exon 29 of the feline PKD1 gene (PKD1 mutation). Many clinical cases have been recognized in Japan, but the mutation rate in cats has not been reported. The objective of this study was to determine epidemiological characteristics and clinical features in cats with the PKD1 mutation. Referring veterinarians sent blood samples of 377 cats for the PKD1 gene evaluation. The blood samples were from 159 cats with renal cysts confirmed by ultrasonography, 60 cats without renal cysts, and 158 cats that did not undergo ultrasonography. In total, 150 cats carried the PKD1 mutation and the signalment, site and number of renal cysts, and results of blood test were evaluated in cats with the PKD1 mutation. The breeds with the highest rate of the PKD1 mutation were Persian (46\%), Scottish Fold (54\%) and American Shorthair cats (47\%). However, mixed breed cats also showed high rates of the PKD1 mutation. Of cats with the mutation, the incidence of high plasma creatinine $(\geq 1.6 \mathrm{mg} / \mathrm{d} /)$ was greater in cats $\geq 3$ years old, although a few cats $\geq 9$ years of age had low plasma creatinine $(<1.6 \mathrm{mg} / \mathrm{d} /)$. The coincidence of renal and hepatic cysts was $12.6 \%$, with the high prevalence in Persian cats (31\%).
\end{abstract}

KEY WORDS: epidemiology, feline polycystic kidney disease, hepatic cysts, PKD1 mutation

Feline polycystic kidney disease (PKD) is an inherited autosomal dominant disease characterized by plural cysts formation in the bilateral kidneys and occasionally in the liver and pancreas [9]. The enlargement of these cysts leads to the development of chronic kidney disease (CKD). The various sized cysts are observed in the renal cortex and medulla of affected cats. The formation and growth of cysts progress slowly, and the renal function gradually deteriorates in the PKD affected cats. The clinical and morphological features of feline PKD are very similar to those of human autosomal dominant polycystic kidney disease (ADPKD) [15]. Therefore, it has been suggested that feline PKD represents a good model for human ADPKD.

Feline PKD was first identified in Persian cats and Persian related breeds [4, 25]. Subsequent studies [21] revealed that a mutation from $\mathrm{C}$ to A at position 10063 in exon 29 of the feline PKD1 gene (PKD1 mutation), which results in a premature stop codon in the mRNA, is the specific cause of PKD. The accumulation of clinical researches on feline PKD revealed that there are many PKD affected cats all over the world. It seems that this disease also spread among the cats of Japan.

Ultrasonographic studies have indicated that the prevalence of PKD in Persian and related cats is about $49.2 \%$ in the United Kingdom [10], 43\% in Australia [2], and 41.8\% in France [3]. Ultrasonography is a good tool for the diagnosis of cystic kidneys. However, PKD is not the only cause of cyst formation in kidneys. Many pathogenic factors such as end stage kidney of chronic nephritis and abnormal condition caused by urinary tract obstruction are involved. Furthermore, kidney diseases that cause pelvic dilation is occasionally misdiagnosed as PKD based on ultrasonography. Therefore, the definitive diagnosis of PKD should be established by genetic testing of the PKD1 mutation. Although the surveys of cats with PKD diagnosed by genetic testing have been conducted in several countries, the number of studied cats was limited [14, 18, 20,21], and little information is available on cats carrying the PKD1 mutation in Japan.

Recent reports [20] have described PKD in feline breeds other than Persians. We previously reported the clinical course 
and cystic fluid characteristics in a Japanese Domestic Shorthair cat with PKD [24]. It is clinically important to investigate the distribution of feline PKD in other feline breeds of Japan because the cats with the PKD1 mutation should not be used for breeding. The aims of this study were to investigate the epidemiological characteristics and clinical features of cats with the PKD1 mutation and to evaluate the prevalence of PKD in Japan.

\section{MATERIALS AND METHODS}

\section{Samples}

Heparinized blood samples that were sent to Iwate University Veterinary Teaching Hospital for testing the PKD1 mutation from 2008 to 2015 were used. These samples were collected from referring veterinary clinics located throughout Japan. Information on these cats was obtained from referring veterinarians, including breed, age, sex, and, if available, the presence, location, and number of renal and hepatic cysts, and plasma concentrations of blood urea nitrogen (BUN), creatinine (Cre), and inorganic phosphate (iP). In some cases, ultrasonographic images of kidneys were obtained from the referring veterinarians and the cystic lesions were confirmed.

\section{Information of animals}

In this study, 377 samples were obtained from cats aged from 0 to 18 years old, and included 166 males, 179 females, and 32 of unknown sex. The breeds included Persian ( $n=79)$, Exotic Shorthair ( $n=71)$, Scottish Fold ( $n=69)$, American Shorthair $(n=34)$, Japanese-mixed $(n=48)$ and the other western-mixed breed cats $(n=11)$. Samples were sent from Hokkaido, Tohoku (Aomori, Iwate, Fukushima), Kanto (Ibaraki, Kanagawa, Saitama, Tochigi, Tokyo, Chiba), Chubu (Niigata, Aichi), Kinki (Osaka, Hyogo, Nara, Shiga, Kyoto), Chugoku (Hiroshima, Shimane), and Kyushu (Kagoshima, Kumamoto) districts.

Of 377 cats examined, 219 cats received ultrasonographic examinations by the referring veterinarians, and 159 of these were reported to have renal cysts and 60 cats were reported to have no cysts. The remaining 158 cats had not undergone ultrasonographic examination. All of the cats were clinically or genetically suspected of PKD by the referring veterinarians, because of abnormal ultrasonography or because they were relatives of PKD affected cats (Persian cats and other breeds including mixed breed are contained).

\section{Genetic testing}

PCR restriction fragment length polymorphism (RFLP) assay was performed on all 377 blood samples at the Department of Veterinary Internal Medicine of Iwate University. Genomic DNA was isolated from heparinized whole blood using a DNA purification kit (DNeasy Blood and Tissue Kit, Qiagen, Hilden, Germany). Exon 29 of the feline PKD1 was partially amplified by PCR, producing a 559-bp amplicon, according to the Lyons et al. [20] protocol. DNA restriction was performed by digestion of 5 $\mu l$ of amplification product in a total reaction volume of $10 \mu l$ that contained $0.5 \mu l$ of FastDigest MLyl (Fermentas, Waltham, MA, U.S.A.) and $10 \times$ FastDigest buffer (Fermentas) incubated at $37^{\circ} \mathrm{C}$ for $30 \mathrm{~min}$, followed by inactivation of enzyme at $80^{\circ} \mathrm{C}$ for 5 min. The RFLP pattern was obtained by running the complete digestion reaction on a $\%$ agarose gel.

\section{Statistical evaluation}

The rate $(\%)$ of the PKD1 mutation [(number of mutation positive cats / total number of cats) $\times 100]$ was calculated for each breed. The sex difference in cats with the PKD1 mutation and in cats with coinciding hepatic cysts was evaluated by $\chi^{2}$ test.

\section{RESULTS}

\section{Results of genetic testing in relation to the presence of renal cysts}

The results of genetic testing in relation to the presence of renal cysts are listed in Table 1. In total, 150 of 377 (40\%) cats carried the PKD1 mutation; of these, 126 (84\%) cats were diagnosed as having renal cysts by ultrasonography, 3 (2\%) had no renal cysts, and in $21(14 \%)$, the presence of renal cysts was unknown because the cats did not undergo ultrasonography.

\section{The PKD1 mutation rate in relation to the signalment of cats}

The results of genetic testing by breed are shown in Table 2. The PKD1 mutation rate was 46\% (36/79) in Persian cats. Other breeds with a high mutation rate $(\geq 40 \%)$ were Scottish Fold, American Shorthair, Maine Coon, Japanese mixed breed, and other western mixed breed cats. In terms of sex difference, the rate of the PKD1 mutation was 46\% (77/166) in males and 37\% (66/179)

Table 1. The result of genetic test in relation to the presence of renal cysts

\begin{tabular}{|c|c|c|c|c|c|c|}
\hline \multirow{3}{*}{$\begin{array}{l}\text { Renal cysts detected by ultrasonography } \\
\text { The number of cats } \\
\text { PKD1 gene mutation }\end{array}$} & \multicolumn{2}{|c|}{ Present } & \multicolumn{2}{|c|}{ Absent } & \multicolumn{2}{|c|}{ Not examined } \\
\hline & \multicolumn{2}{|c|}{159} & \multicolumn{2}{|c|}{60} & \multicolumn{2}{|c|}{158} \\
\hline & Positive & Negative & Positive & Negative & Positive & Negative \\
\hline The number of cats $(\%)$ & $126(79 \%)^{\mathrm{a})}$ & $33(21 \%)$ & $3(5 \%)^{a)}$ & $57(95 \%)$ & $21(13 \%)^{\mathrm{a})}$ & $137(87 \%)$ \\
\hline
\end{tabular}

a) Total number of positive cats is $150(40 \%)$. 
Table 2. The results of genetic test in relation to the breeds of cats

\begin{tabular}{lcccc}
\hline \multicolumn{1}{c}{ Breed } & Positive (n) & Negative (n) & Positive rate (\%) & Total number \\
\hline Persian cats & 36 & 43 & 46 & 79 \\
Exotic Shorthair & 16 & 55 & 23 & 71 \\
Scottish Fold & 37 & 32 & 54 & 69 \\
American Shorthair & 16 & 18 & 47 & 34 \\
Munchkin & 6 & 10 & 38 & 16 \\
Maine Coon & 2 & 2 & 50 & 4 \\
Japanese mixed breed & 26 & 22 & 54 & 48 \\
Other western mixed breed & 8 & 3 & 73 & 11 \\
\hline
\end{tabular}

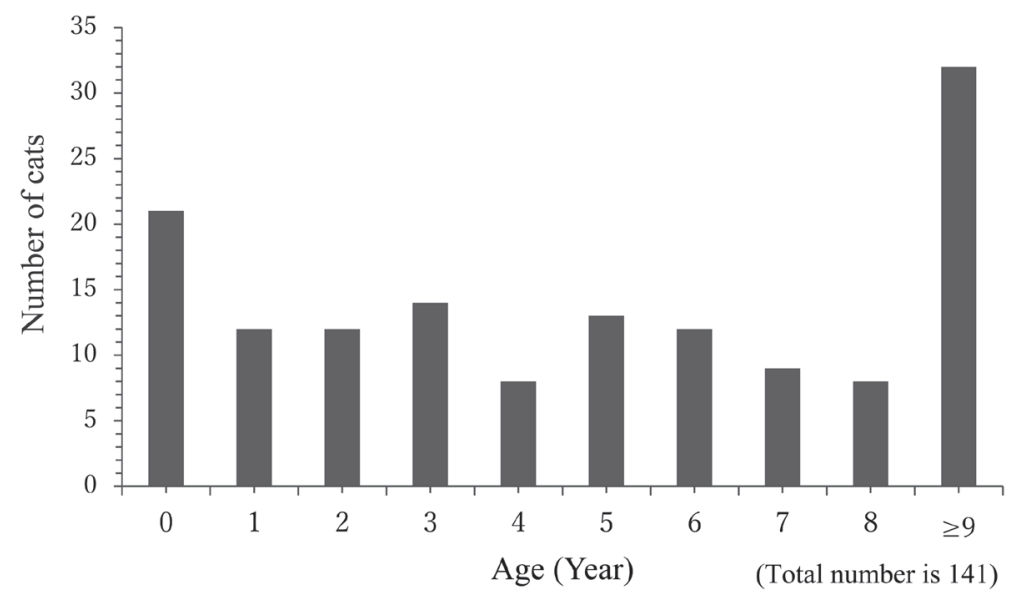

Fig. 1. The age distribution of cats with the PKDl mutation.

in females; the sex of 32 cats was unknown. The mutation rate did not differ significantly between male and female cats by $\chi^{2}$ test $(P=0.357)$. The age distribution of cats with the $P K D 1$ mutation is shown in Fig. 1; the cats ranged from $>1$ to 18 years old; the average $( \pm \mathrm{SD})$ age was $5.4 \pm 4.5$ years old.

\section{Evaluation of renal function in cats with the PKD1 mutation}

One hundred and two cats with the PKD1 mutation also had data on blood Cre concentration. The relationship of blood Cre concentration with age is shown in Fig. 2. Of cats with the PKD1 mutation, the incidence of a high concentration of plasma Cre $(>1.6 \mathrm{mg} / \mathrm{d} l$ : $\geq$ IRIS-CKD stage 2$)$ was greater in cats older than 3 years old, and especially in those older than 7 years. In contrast, a few cats aged $\geq 9$ years had low plasma Cre concentrations $(\leq 1.6 \mathrm{mg} / \mathrm{d} l)$.

\section{Coincidence of renal and hepatic cysts}

The association between renal and hepatic cysts was also investigated. Co-occurrence of hepatic and renal cysts was found in 20 (12.6\%) out of 159 cases with renal cyst(s), and all of these cases (13 males, 6 females, and 1 cat of unknown sex, 2 to 17 years old: $5.2 \pm 4.4$ years) were positive for the $P K D 1$ mutation. A significant difference in the $P K D 1$ mutation rate between male and female cats was not detected by $\chi^{2}$ test $(P=0.44)$. The prevalence of co-occurrence of renal and hepatic cysts by breed is shown in Table 3. Of note, Persian cats were overrepresented: $31 \%$ of all Persian cats examined had co-occurring renal and hepatic cysts, and a few cats of other breeds, including Scottish Fold and American Shorthair cats, had co-occurring cysts.

\section{DISCUSSION}

Since 2000, the prevalence of feline PKD have been investigated in several countries, including the United States, Italy, the United Kingdom, Australia, France, Slovenia and Taiwan [2, 3, 5, 10, 14, 20]. Many reports have identified the breeds at high risk for PKD, including Persians and Persian related breeds $[2,5,6,12,15]$. Since 2004, reports on the diagnosis of PKD using genetic testing have increased, and the PKD1 mutation rate is reported to be $15.7 \%$ in Persian cats in Taiwan [20], 33.3\% in Persian cats in Slovenia [14], and 37.1\% in Persian cats and Exotic Shorthairs in Italy [8]. In our study, the PKD1 mutation rate in Persian cats was $46 \%$. When considering the breeding bias in previous studies and the present study, this rate is higher than previously reported. However, the samples used in this study were obtained from cases in which renal cysts were detected by ultrasonography, or from those related to cats with PKD, or from high risk breeds. Therefore, the true prevalence of the PKD1 mutation in Persian cats in 


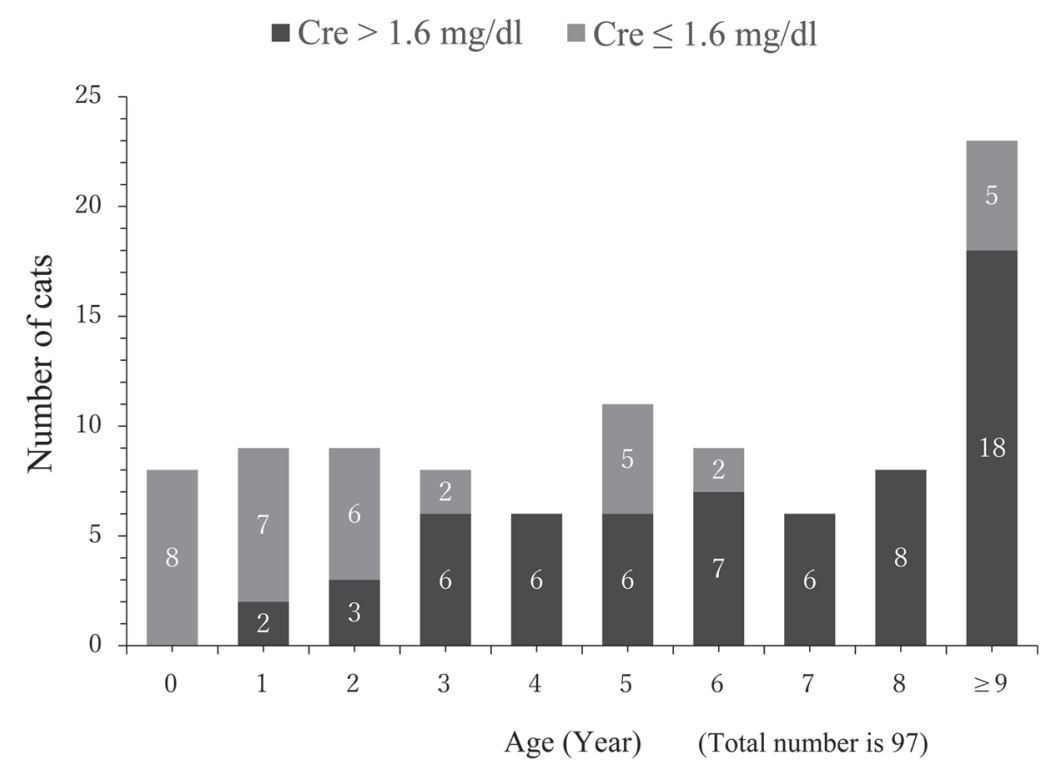

Fig. 2. The relationship between blood creatinine concentration and the age of cats with the PKD1 mutation.

Table 3. The breeds of cats complicated with renal and hepatic cysts

\begin{tabular}{lcc}
\hline \multicolumn{1}{c}{ Breed } & $\begin{array}{c}\text { Case number } \\
\text { (Number of male cats) }\end{array}$ & $\begin{array}{c}\text { Prevalence } \\
(\%)^{\mathrm{a})}\end{array}$ \\
\hline Persian cats & $11(7)$ & 31 \\
Scottish Fold & $3(1)$ & 16 \\
Amerian Shorthair & $2(2)$ & 12 \\
Other western-mixed breed & 1 (unknown) & 6 \\
Japanese-mixed breed & $2(2)$ & 12 \\
Unkown & $1(1)$ & 5 \\
\hline Total & $20(13)$ & \\
\hline
\end{tabular}

a) The prevalence in each cat breed.

Japan is expected to be lower than that reported in the current study.

In recent reports from other countries, Persian related breeds such as Exotic Shorthair and British Shorthair are also at high risk for PKD, and the Bombay and Ragdoll breeds are at moderate risk [3, 23]. Polycystic kidney disease has been found in other breeds, including American Shorthair, Chinchilla, Irish Shorthair and Exotic Shorthair [20]. However, the incidence in these breeds is much lower than in Persian cats. Several studies from various parts of the world have reported that the prevalence of PKD detected by ultrasonography ranged from 37 to $49 \%$ in Persian and Persian related cats and was around $16 \%$ in other breeds [2, $3,10]$. In the present study, it was found that Scottish Fold and American Shorthair cats also had high rates in the PKD1 mutation (a few cases were reported in previous studies). These results suggest that the disease is distributed among many breeds of cat in Japan. In addition, cats with the PKD1 mutation may be blindly used for breeding with cats of other breeds.

In the present study, results of genetic evaluation were inconsistent with cyst formation detected by ultrasonography. Thirtythree cats with renal cysts detected by the referring veterinarians using ultrasonography showed negative by genetic testing for the PKD1 mutation. Although renal cysts in PKD are detected easily by ultrasonography, hydronephrosis with dilated renal pelvis or renal cysts formed by dilation of urinary tubules in late stage of chronic nephritis can be mistaken as cysts of PKD [19]. Indeed, some reports have described cats of the Maine Coon breed in which renal cysts formed as a result of chronic nephritis but that did not have the PKD1 mutation [18]. The results of ultrasonography often depend on the type of probe, the equipment used, and the technical skill of the operator. When we reviewed the ultrasonographic images sent by the referring veterinarians, many images were not consistent with cysts of PKD, whereas 3 cats diagnosed as having no cyst by the referring veterinarians were positive for the PKD1 mutation. Two of these cats were very young ( $<1$ years old) and the age of the third cat was unknown. It is difficult to investigate the renal cysts in very young cats, especially those under 4 months old.

However, the possibility that another mutation exists at an unknown location cannot be excluded. The $P K D 1$ gene is very large and has 46 exons. In human ADPKD, it is known that the disease can be caused by mutations in the PKD1 or PKD2 gene, and nonsense or frameshifting mutation have been identified at various positions [22, 26]. Therefore, analysis of mutations at other positions in the feline $P K D 1$ and $P K D 2$ genes may also be needed in the future. 
One clinical sign of PKD is an elevated serum Cre concentration. When cats with a high Cre concentration (corresponding to IRIS-CKD stageII or higher) were evaluated, the proportion tended to increase from 3 years old, and especially in those older than 7 years of age. However, some cats aged 9 or more had normal Cre concentration. These results suggested that clinical course in PKD is variable; in some cases, the disease progresses rapidly and results in azotemia, and in other cases, the disease progresses slowly, and renal function remains normal for a long time. In human ADPKD, the growth rate of cysts varies among patients and depends on the mutation position $[11,26]$. In feline PKD, the disease-causing mutation is identified at only one position but, as described above, the possibility of mutation at other locations cannot be excluded. Recently, a "third hit hypothesis" was proposed to explain the pathogenesis in human ADPKD. In this hypothesis, the formation of cysts is hastened by presence of other complications affecting the renal parenchyma or function. Further investigation is required to clarify whether concurrent diseases, such as urolithiasis or urinary tract infection for example, affect cyst formation in PKD affected cats.

Hepatic cyst is an extrarenal manifestations that occurs in a few cases of PKD [9]. In humans with ADPKD, the rate of coincident renal and hepatic cysts is about $80 \%$ [1], whereas a few studies have reported coincident renal and hepatic cysts in cats $[7,9]$. According to the research in Italy [7], 8 of $136(5.9 \%)$ animals with renal cysts had one or more hepatic cysts concurrently; all of the cats were male Persian cats, but genetic testing was not performed. In our study, the rate of coincident renal and hepatic cysts was $12.6 \%$, and all cases were positive for the PKD1 mutation. Although not significantly different, more male than female cats were affected with coincident renal and hepatic cysts in the present study, which is similar to previous reports [7]. In addition, the incidence of hepatic cysts in PKD and the high prevalence in Persian cats in our study are consistent with these reports. The high incidence of hepatic cysts in Persian cats suggests that the incidence may differ among breeds. In human ADPKD, the incidence of hepatic cysts is higher in women, so this condition is thought to be related with pregnancy or female hormone [1, 16, 17]. In addition, hepatic cysts occurring in human ADPKD are thought to represent marked dilation of bile ducts in bile-duct hamartomas [13]. Pathological analysis of hepatic cysts in feline PKD shows that the cysts are lined with flattened epithelial cells and are not associated with other hepatobiliary lesions [15]. Therefore, hepatic cysts in feline PKD may have a different pathogenesis from those in human ADPKD, and other factors might be associated with this lesion in cats. Further investigation is required to determine the pathogenesis of hepatic cysts in feline PKD. Furthermore, there was no significant difference in age between cats with hepatic cysts and those without. Therefore, disease stage may not be related to hepatic cyst formation.

This study revealed the epidemiological features of the cats with the PKD1 mutation in a large population of the cats suspected to have ADPKD. In contrast to results from other countries, the cats with the PKD1 mutation are widely distributed in Japan, not only in Persian cats but also in non-Persians. Affected cats tend to develop signs of impaired renal function from as early as 3 years of age, and the clinical course can vary. In addition, relatively high coincidence of renal and hepatic cysts in the cats with the PKD1 mutation was found. It is strongly recommended that cats positive for the PKD1 mutation are not used for breeding.

\section{REFERENCES}

1. Bae, K. T., Zhu, F., Chapman, A. B., Torres, V. E., Grantham, J. J., Guay-Woodford, L. M., Baumgarten, D. A., King, B. F. Jr., Wetzel, L. H., Kenney, P. J., Brummer, M. E., Bennett, W. M., Klahr, S., Meyers, C. M., Zhang, X., Thompson, P. A., Miller J. P., Consortium for Radiologic Imaging Studies of Polycystic Kidney Disease (CRISP). 2006. Magnetic resonance imaging evaluation of hepatic cysts in early autosomal-dominant polycystic kidney disease: the consortium for radiologic imaging studies of polycystic kidney disease cohort. Clin. J. Am. Soc. Nephrol. 1: 64-69. [Medline] [CrossRef]

2. Barrs, V. R., Gunew, M., Foster, S. F., Beatty, J. A. and Malik, R. 2001. Prevalence of autosomal dominant polycystic kidney disease in Persian cats and related-breeds in Sydney and Brisbane. Aust. Vet. J. 79: 257-259. [Medline] [CrossRef]

3. Barthez, P. Y., Rivier, P. and Begon, D. 2003. Prevalence of polycystic kidney disease in Persian and Persian related cats in France. J. Feline Med. Surg. 5: 345-347. [Medline] [CrossRef]

4. Battershell, D. and Garcia, J. P. 1969. Polycystic kidney in a cat. J. Am. Vet. Med. Assoc. 154: 665-666. [Medline]

5. Beck, C. and Lavelle, R. B. 2001. Feline polycystic kidney disease in Persian and other cats: a prospective study using ultrasonography. Aust. Vet. J. 79: 181-184. [Medline] [CrossRef]

6. Biller, D. S., DiBartola, S. P., Eaton, K. A., Pflueger, S., Wellman, M. L. and Radin, M. J. 1996. Inheritance of polycystic kidney disease in Persian cats. J. Hered. 87: 1-5. [Medline] [CrossRef]

7. Bonazzi, M., Volta, A., Gnudi, G., Bottarelli, E., Gazzola, M. and Bertoni, G. 2007. Prevalence of the polycystic kidney disease and renal and urinary bladder ultrasonographic abnormalities in Persian and Exotic Shorthair cats in Italy. J. Feline Med. Surg. 9: 387-391. [Medline] [CrossRef]

8. Bonazzi, M., Volta, A., Gnudi, G., Cozzi, M. C., Strillacci, M. G., Polli, M., Longeri, M., Manfredi, S. and Bertoni, G. 2009. Comparison between ultrasound and genetic testing for the early diagnosis of polycystic kidney disease in Persian and Exotic Shorthair cats. J. Feline Med. Surg. 11: 430-434. [Medline] [CrossRef]

9. Bosje, J. T., van den Ingh, T. S. and van der Linde-Sipman, J. S. 1998. Polycystic kidney and liver disease in cats. Vet. Q. 20: 136-139. [Medline] [CrossRef]

10. Cannon, M. J., MacKay, A. D., Barr, F. J., Rudorf, H., Bradley, K. J. and Gruffydd-Jones, T. J. 2001. Prevalence of polycystic kidney disease in Persian cats in the United Kingdom. Vet. Rec. 149: 409-411. [Medline] [CrossRef]

11. Cavenee, W. K., Dryja, T. P., Phillips, R. A., Benedict, W. F., Godbout, R., Gallie, B. L., Murphree, A. L., Strong, L. C. and White, R. L. 1983. Expression of recessive alleles by chromosomal mechanisms in retinoblastoma. Nature 305: 779-784. [Medline] [CrossRef]

12. Cooper, B. K. and Piveral, P. 2000. Autosomal dominant polycystic kidney disease in Persian cats. Feline Pract. 28: 20-21.

13. Desmet, V. J. 1992. Congenital diseases of intrahepatic bile ducts: variations on the theme "ductal plate malformation". Hepatology 16: 1069-1083. [Medline] [CrossRef]

14. Domanjko-Petric, A., Cernec, D. and Cotman, M. 2008. Polycystic kidney disease: a review and occurrence in Slovenia with comparison between ultrasound and genetic testing. J. Feline Med. Surg. 10: 115-119. [Medline] [CrossRef]

15. Eaton, K. A., Biller, D. S., DiBartola, S. P., Radin, M. J. and Wellman, M. L. 1997. Autosomal dominant polycystic kidney disease in Persian and 
Persian-cross cats. Vet. Pathol. 34: 117-126. [Medline] [CrossRef]

16. Everson, G. T. 1993. Hepatic cysts in autosomal dominant polycystic kidney disease. Am. J. Kidney Dis. 22: 520-525. [Medline] [CrossRef]

17. Gabow, P. A., Johnson, A. M., Kaehny, W. D., Manco-Johnson, M. L., Duley, I. T. and Everson, G. T. 1990. Risk factors for the development of hepatic cysts in autosomal dominant polycystic kidney disease. Hepatology 11: 1033-1037. [Medline] [CrossRef]

18. Gendron, K., Owczarek-Lipska, M., Lang, J. and Leeb, T. 2013. Maine Coon renal screening: ultrasonographical characterisation and preliminary genetic analysis for common genes in cats with renal cysts. J. Feline Med. Surg. 15: 1079-1085. [Medline] [CrossRef]

19. Helps, C., Tasker, S. and Harley, R. 2007. Correlation of the feline PKD1 genetic mutation with cases of PKD diagnosed by pathological examination. Exp. Mol. Pathol. 83: 264-268. [Medline] [CrossRef]

20. Lee, Y. J., Chen, H. Y., Hsu, W. L., Ou, C. M. and Wong, M. L. 2010. Diagnosis of feline polycystic kidney disease by a combination of ultrasonographic examination and PKD1 gene analysis. Vet. Rec. 167: 614-618. [Medline] [CrossRef]

21. Lyons, L. A., Biller, D. S., Erdman, C. A., Lipinski, M. J., Young, A. E., Roe, B. A., Qin, B. and Grahn, R. A. 2004. Feline polycystic kidney disease mutation identified in PKD1. J. Am. Soc. Nephrol. 15: 2548-2555. [Medline] [CrossRef]

22. Magistroni, R., He, N., Wang, K., Andrew, R., Johnson, A., Gabow, P., Dicks, E., Parfrey, P., Torra, R., San-Millan, J. L., Coto, E., Van Dijk, M., Breuning, M., Peters, D., Bogdanova, N., Ligabue, G., Albertazzi, A., Hateboer, N., Demetriou, K., Pierides, A., Deltas, C., St George-Hyslop, P., Ravine, D. and Pei, Y. 2003. Genotype-renal function correlation in type 2 autosomal dominant polycystic kidney disease. J. Am. Soc. Nephrol. 14: 1164-1174. [Medline] [CrossRef]

23. Margie, S. 2014. Feline Polycystic Kidney Disease. Clinician's Brief 12: 79-82.

24. Sato, R., Kobayashi, S., Sasaki, K., Uto, W., Goryo, M., Sasaki, J., Kamishina, H., Oishi, A. and Yasuda, J. 2010. Change of renal cystic fluid in a cat with feline polycystic kidney disease caused by PKD-1 gene mutation. Nippon Juishikai Zasshi 63: 791-796.

25. Silvestro, D. 1967. [On a case of bilateral polycystic kidney in a cat]. Acta Med. Vet. (Napoli) 13: 349-361 (in Italian). [Medline]

26. Qian, F. and Germino, G. G. 1997. "Mistakes happen": somatic mutation and disease. Am. J. Hum. Genet. 61: 1000-1005. [Medline] [CrossRef] 